\title{
Assessment of the COVID-19 infection risk at a workplace through stochastic microexposure modeling
}

\author{
Sergey Vecherin ${ }^{1 凶}$, Derek Chang ${ }^{1}$, Emily Wells ${ }^{1,2}$, Benjamin Trump ${ }^{1}$, Aaron Meyer ${ }^{1}$, Jacob Desmond ${ }^{1}$, Kyle Dunn ${ }^{1}$, Maxim Kitsak ${ }^{3}$ and \\ lgor Linkov (iD ${ }^{1,2}$ 凶
}

This is a U.S. government work and not under copyright protection in the U.S.; foreign copyright protection may apply 2022

BACKGROUND: The COVID-19 pandemic has a significant impact on economy. Decisions regarding the reopening of businesses should account for infection risks.

OBJECTIVE: This paper describes a novel model for COVID-19 infection risks and policy evaluations.

METHODS: The model combines the best principles of the agent-based, microexposure, and probabilistic modeling approaches. It takes into account specifics of a workplace, mask efficiency, and daily routines of employees, but does not require specific interagent rules for simulations. Likewise, it does not require knowledge of microscopic disease related parameters. Instead, the risk of infection is aggregated into the probability of infection, which depends on the duration and distance of every contact. The probability of infection at the end of a workday is found using rigorous probabilistic rules. Unlike previous models, this approach requires only a few reference data points for calibration, which are more easily collected via empirical studies.

RESULTS: The application of the model is demonstrated for a typical office environment and for a real-world case.

CONCLUSION: The proposed model allows for effective risk assessment and policy evaluation when there are large uncertainties about the disease, making it particularly suitable for COVID-19 risk assessments.

Keywords: Disease Spreading Modeling; Microenvironment approach; Probability of infection; Spatial probability of transmission; Temporal probability of transmission; COVID-19 risk assessment

Journal of Exposure Science \& Environmental Epidemiology (2022) 32:712-719; https://doi.org/10.1038/s41370-022-00411-2

\section{INTRODUCTION}

The COVID-19 pandemic has caused severe disruption to our normal lives [1]. The pandemic has imposed a severe toll on human health and wellbeing, destroyed families, and significantly affected the economy $[2,3]$. COVID-19's impact to the economy in the long run can be devastating. It is apparent that lockdown of all businesses can be used as a short-term emergency policy, but will not be viable or sustainable over prolonged periods for many businesses [4]. Therefore, risk infection models are needed to make decisions regarding workplace opening and pandemic mitigation policies [5].

There are several approaches for modeling the spread of viral infections. One of the most renowned approach is the susceptibleinfected-recovered (SIR) models [6-8]. In fact, ref. [9] cites the CHIME model [10], which is a SIR model with a one-day cycle, as one of the most widely used tools in the US. In this approach, the main assumption is that the population is homogeneous and flows between different categories at rates specific to the disease being modeled. Typically, these models are deterministic, described by a system of differential equations with fixed rates of transition from one category to the next. Additional models have incorporated uncertainty in the parameter estimation [11] and time-varying parameters [12]. Although these models require relatively few parameters, one must provide rates of infection for individuals who come into contact with one another. At small scales and heterogeneous population, these rates can vary drastically from one individual to the next depending on effective precautions taken and individual's daily routine at the workplace. Furthermore, significant complexity arises when a specific workplace floorplan is considered, or when local mitigation factors are imposed on a workforce, such as social distancing, or staggered scheduling [13].

The agent-based models for COVID-19 can overcome many of the shortcomings of the SIR models $[14,15]$. In this approach, relatively simple rules are prescribed for every agent (e.g., a person) to model its behavior. The simulation then evolves in time according to these interaction rules. Eventually, the collective outcome of such interactions can be analyzed on the macro level to make meaningful statistical inferences. These models can capture many individual-level behaviors, but at the risk of being too detailed. Among known issues with agentbased modeling are the dependence of the results on initial conditions (i.e., it will matter which specific employees are infected at the beginning of the simulation, not only how many) and on the internal structure of the model itself, so that simulations with the same parameters lead to different

\footnotetext{
${ }^{1}$ Engineer Research and Development Center, Vicksburg, MS, USA. ${ }^{2}$ Carnegie Mellon University, Pittsburgh, PA, USA. ${ }^{3}$ Delft University of Technology, Delft, Netherlands.
}

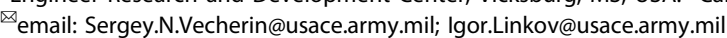


inferences. Another issue is the difficulty in calibration of the rules between the agents so that the macro effect would match the empirical knowledge $[16,17]$.

Another established approach for disease spread modeling is based on aerosol simulation techniques $[18,19]$. This approach assumes that infection occurs when a number of contaminants in the air exceeds a certain concentration. The approach can incorporate the specific building design, air paths, and ventilation systems to estimate pathogen concentrations, and, thus, make an inference on the infection spread. Some of the simulations include a full fluid dynamic solution for air motion in a specific floorplan of the building [20]. However, the aerosol mechanism of COVID-19 transmission, unlike the close-contact transmission, is debatable, and, currently, there is no expert agreement on whether this mechanism is actually engaged [21]. Furthermore, aerosol models require very specific infection parameters as inputs, such as the virus emission rate, the size distribution of aerosol particles that carry viable viruses, aerosol viral load, and the minimum infectious dose. None of these parameters are known for COVID-19 exactly. Furthermore, other characteristics are required, such as lung capacity, speech/breath ratio, and concentration of infectious particles at an exhale, which can vary by orders of magnitude among different individuals, and depend on the surrounding conditions and ventilation efficiency [18]. In this paper, the aerosol transmission mechanism is not considered in the model, but the model could be modified to include this or other mechanisms.

The stochastic model developed in this paper was derived fusing principles of "microexposure" $[22,23]$ modeling, agentbased modeling, and probabilistic modeling, which together are best suited to estimate individual-level COVID-19 exposure and infection risk. A daily workplace activity of an individual is parsed to elementary typical work environments. We estimate the probability of becoming infected in each of these microenvironments. Then, fusing probabilities of all activities for the entire day, we obtained the probability that a single uninfected person becomes infected at the end of his or her typical workday.

Similar to the agent-based and microexposure models, MonteCarlo simulations are used in our model to estimate the number and duration of an uninfected individual's encounters with others. However, for each single interaction, the quantity of interest is the probability of becoming infected, which depends on the closest distance and duration of the contact. The innovation in comparison with other approaches is that the infection probability is modeled directly by introducing explicit formulas for the spatialtemporal probability of virus contraction. These probabilities require only a few parameters for calibration that can be estimated from empirical data easier than collecting precise information about virus concentration and person's lung capacity. Once the probability is estimated, the fusion of different microenvironment risks can be implemented rigorously using the theory of probability. This significantly reduces the required number of trials in the Monte-Carlo simulations and removes oversensitivity of the agent-based models to the initial conditions.

The proposed model can be used in practice in three different ways. The main application is to predict disease dynamics in time for a specific workplace. For this purpose, all or some parameters of the model either should be estimated by direct measurements at that workplace, or should be treated as fitting parameters to match the predictions to actual new cases during the few first days observed at that workplace. Another application is to predict a typical-case infection dynamics at a desired scale. For this application, typical statistical parameters can be used. And finally, the model can be used to estimate relative effectiveness of workplace policies aimed at preventing the spread of the disease at a workplace. In the paper, examples for all three model applications are provided.

\section{MODEL}

\section{Baseline micro model}

In the baseline micro model, the probability of becoming infected due to a single contact between two people is deemed dependent on three probabilities: the probability that a randomly chosen person is infected, the probability that an infected person exposes another person to the virus, and the probability of contracting the virus. Specifically, the single contact infection probability is described by the following formula:

$P_{\mathrm{j}, \text { inf }}=P_{0} P_{\exp , a_{i}} P_{c, a_{j}}(r, t)$,

where $P_{\mathrm{j}, \text { inf }}$ is the probability that an uninfected person $j$ becomes infected after a single contact with a person $i, P_{0}$ is the probability that a randomly chosen person $i$ is infected, $P_{\text {exp, } a_{i}}$ is the probability of virus exposure, $P_{c . a_{j}}$ is the probability of virus contraction, $a_{i}$ and $a_{j}$ are the ith and jth person's behavioral archetypes (explained below), $r$ is the closest distance of contact between the person $i$ and $j$, and $t$ is the duration of the contact at the closest distance.

The probability of virus exposure, $P_{\exp , a_{i}}$, characterizes the effectiveness of spreading the virus by an infected person $i$ and depends on the infected person's behavioral archetype, $a_{i}$. The behavioral archetype reflects usage of different personal protection equipment (PPE) or adherence to other safety compliance measures. For simplicity, let $a_{i}$ denote a random event of the $i$ th person wearing a mask and $\bar{a}_{i}$ denote the $i$ th person not wearing a mask. Then, $P_{\text {exp }, a_{i}}$ can be expressed as:

$P_{\exp , a_{i}}=P\left(\exp \mid a_{i}\right) P_{a_{i}}+P\left(\exp \mid \bar{a}_{i}\right)\left(1-P_{a_{i}}\right)$

where $P\left(\exp \mid a_{i}\right)$ is the probability for virus exposure under the condition that the ith person wears a mask, $P\left(\exp \mid \bar{a}_{i}\right)$ is the probability for virus exposure under the condition that the $i$ th person does not wear a mask, and $P_{a_{i}}$ is the probability that the ith person wears a mask. These probabilities can be treated as fitting parameters at model calibration to a specific community or microenvironment.

The probability that the $j$ th person contracts the virus, $P_{c, a_{j}}(r, t)$, depends on the behavioral archetype of the $j$ th person (i.e., if the jth person wears a mask), the closest distance between the ith and jth individuals, and the duration of the contact:

$P_{c, a_{j}}(r, t)=P_{c, r}\left(a_{j}, r\right) P_{c, t}\left(a_{j}, t\right)$

where $P_{c, r}\left(a_{j, r}\right)$ and $P_{c, t}\left(a_{j}, t\right)$ are spatial and temporal probabilities of virus contraction, both dependent on the jth person's behavioral archetype.

The spatial dependence of the risk of virus contraction may not be known exactly. Currently, there is no unanimous agreement on which functional form for spatial dependence is most accurate for modeling COVID-19 risks [24]. Some studies suggest using the inverse squared [24] or exponential models [25]. Moreover, if the aerosol mechanism for COVID-19 spreading is occurring, then the spatial dependence will likely be anisotropic and affected by the airflow in the building. In this paper, we do not pursue this direction and limit ourselves by the isotropic inverse squared model, suitable for the aerial droplet transmission mechanism, following the same arguments presented in ref. [24]:

$$
P_{c, r}\left(a_{j}, r\right)= \begin{cases}\frac{C\left(a_{j}\right)}{r^{2}}, & r \geq R\left(a_{j}\right) \\ 1, & r<R\left(a_{j}\right)\end{cases}
$$

where $C\left(a_{j}\right)$ is a fitting constant to match empirical data for an archetype $a_{j}$, and $R\left(a_{j}\right)$ can be interpreted as a critical distance below which contracting the virus for long exposure times becomes inevitable, i.e., $P_{c, r}\left(a_{j}, R\right)=1$. Keeping in mind that $a_{j}$ denotes the $j$ th individual wearing a mask, and $\bar{a}_{j}$ denotes the 

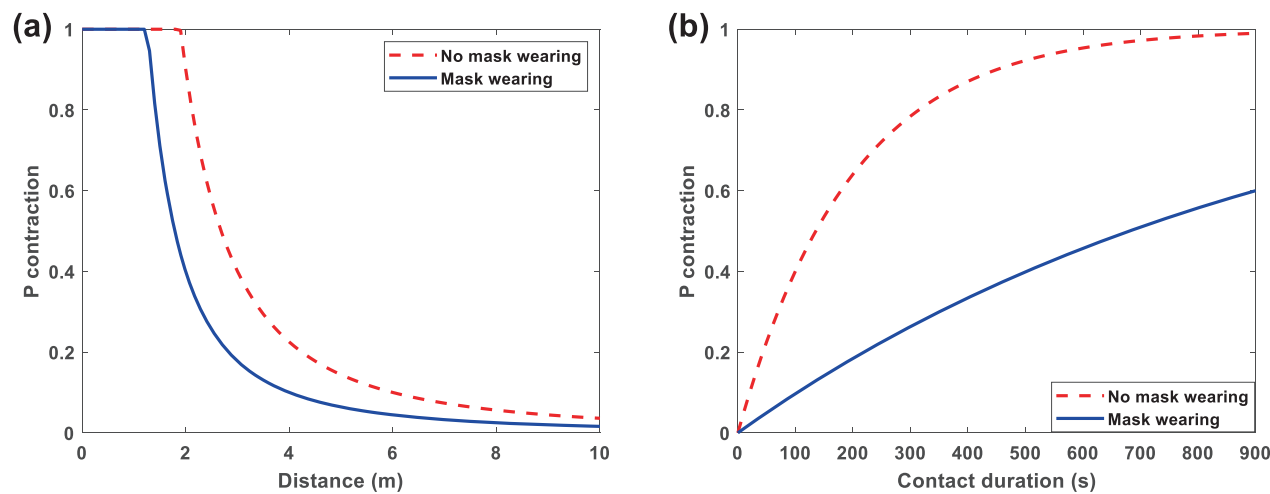

Fig. 1 Probability of virus contraction. a Spatial dependence. b Temporal dependence.

opposite, it is reasonable to set up the following reference point: the probability to contract the virus at $r=2 \mathrm{~m}$ and at infinite exposure time is 0.9 for the $\bar{a}_{j}$ and 0.4 for the $a_{j}$ archetypes. Although 0.9 value for the reference point is chosen arbitrarily, it reflects the current $\mathrm{CDC}$ recommendations stating that the probability of virus contraction is "high" at close distances. The infection risk of 0.4 under mask usage is linked to the choice of 0.9 and is based on particle removal efficiency of masks, which is estimated to be $53-75 \%$ for commercial surgical masks and can reach $90 \%$ when snugged to the face under a nylon layer [26]. These choices result in the following fitting constants from Eq. (4):

$$
C\left(a_{j}\right)=1.6 \mathrm{~m}^{2}, C\left(\bar{a}_{j}\right)=3.6 \mathrm{~m}^{2}, R\left(a_{j}\right)=1.26 \mathrm{~m}, R\left(\bar{a}_{j}\right)=1.90 \mathrm{~m}
$$

The temporal dependence is modeled using the Wells-Riley equation [27], which describes the probability of airborne infection under some specific assumptions. Specifically, it operates in terms of an infection "quanta" and assumes the Poisson distribution of infected particles in the air [28]. The Wells-Riley equation reads as following:

$P_{l}=1-\exp \left(-\frac{l p q t}{Q}\right)$

where $P_{l}$ is the probability of infection, $l$ is the number of infectors, $p$ is the pulmonary ventilation rate of a person, $q$ is the quanta generation rate, $t$ is the exposure time interval, and $Q$ is the room ventilation rate with the clean air. The Wells-Riley equation is applicable to any disease with airborne transmission mechanism (subject to assumptions made in the derivation of the Wells-Riley equation), particularly, it is applicable to COVID-19.

In our paper, we aggregated disease- and person-specific characteristics, $p$ and $q$, and the room ventilation rate $Q$ into a single new variable, called $T=Q / l q p$. The motivation is that it is more practical to estimate a single parameter $T$ from a single reference point than four other parameters (also found from a reference point). As the result, the following equation was used in the manuscript:

$P_{c, t}\left(a_{j}, t\right)=1-\exp \left(-\frac{t}{T\left(a_{j}\right)}\right), t \geq 0$,

where the fitting time constant $T$ depends on the person behavioral archetype $a_{j}$ because all four parameters are affected by wearing a mask, $l, p, q$, and $Q$.

According to the CDC recommendations [29], a 15-min exposure to the virus in close contact with an infected person leads to a high risk of being infected. Setting the probability of contraction during a 15-min contact to 0.99 for the archetype $a_{j}$ and 0.6 for the archetype $\bar{a}_{j}$, the temporal fitting constants are:

$T\left(a_{j}\right)=982.22 \mathrm{~s}, T\left(\bar{a}_{j}\right)=195.43 \mathrm{~s}$

Figure $1 \mathrm{a}, \mathrm{b}$ shows the spatial and temporal probabilities of virus contraction, respectively.

Equation 1 provides a probability of being infected in a single contact. If an uninfected person $j$ experiences $N$ contacts, then, under assumptions that these contacts are statistically independent and identical, the probability of being infected in at least one of the contacts can be found as:

$P_{\text {inf }}(N)=1-\left(1-P_{\mathrm{j}, \text { inf }}\right)^{N}$

where $P_{\mathrm{j}, \mathrm{inf}}$ is given by Eq. 1 .

\section{In-person work meetings}

Work meetings represent one of the typical microenvironments at a workplace. It is assumed that in-person meetings take place in a large room, where people are seated at specific places on a round or rectangular table. The number of participants, time spent in the meetings, dimensions of the tables, and places where the employees are seated can be regulated or measured. The duration of contact is assumed to be the same for any person at the meeting. The distribution of people around both types of tables are different, and because the separation distance between employees is an ingredient in the model, so too are the risks of infection.

Fig. 2a shows the probability of becoming infected during a 60min work meeting held at round tables of different radii, for scenarios where all participants wear a mask, and where no participants wear masks. Note that the size of the table has a more profound effect in the case of not wearing masks than in the case when all participants wear masks. Also of interest to note: masks wearing is a far more dominant effect than the table size, especially for small tables (compare the solid and dotted lines, and the dashed and dash-dotted lines).

Figure $2 \mathrm{~b}$ depicts the probability of becoming infected at a $2 \mathrm{~m}$ radius round table for meetings of different durations, where all participants wear masks. One insight in Fig. $2 b$ is that the probability has uneven time dependence, i.e., it saturates at about 60 -min meetings. Another note of interest is that the dependence on the number of participants bears quadratic, rather than linear, character. The same feature is present in Fig. 2a, but less pronounced due to different axis scale. For closed space scenarios, like having a room or a table of a fixed size, the distance between $N$ people becomes inverse proportional to $N$. Recalling that the spatial probability of contracting the virus is inversely proportional to the distance squared, the squared dependence on $N$ becomes apparent. If any single individual has the same probability, the expected number to become infected in the group of $N$ people 
(a)

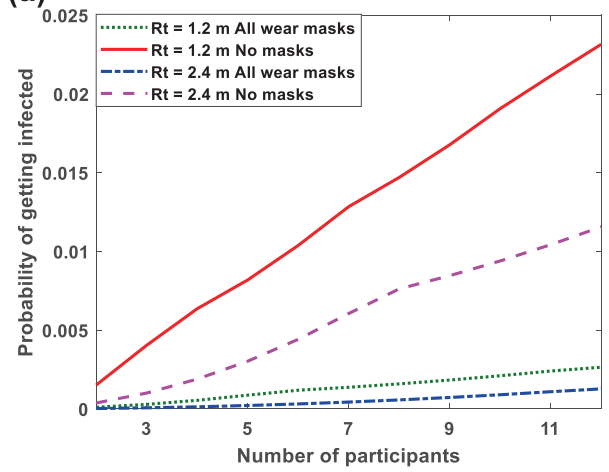

(b)

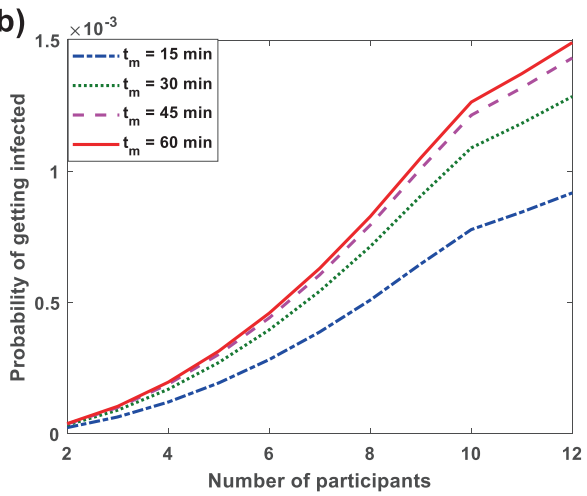

Fig. 2 Probability of becoming infected at meetings at a round table. a 60 -min-long meeting. b $2 \mathrm{~m}$-radius table, all participants wear masks.

can be found as a product of $N$ and the individual probability. Therefore, for close spaces and a group of $N$ people, the average number of attendees getting infected is proportional to $N^{3}$, leading to a "super spreading" event, where a twofold increase in the number of meeting participants leads to an eightfold increase of new infections.

\section{Time dynamics}

The daily infection probability can be used to predict the expected number of infections at the workplace as a function of time. Let the infections occur at the end of the day, and the moment an employee becomes infected, they are both asymptomatic and contagious during the incubatory period $i_{0}$. After this time has ended, they may develop symptoms, which is controlled by the asymptomatic rate parameter $a$. In this case, they quarantine at home for the duration of infection. The employee is infected for a total duration of $\tau$, after which they either recover or die according to the recovery rate $\beta$. Once an employee recovers, they are immune to a second infection. As the number of infected employees in the workplace grows, the probability a particular employee is infected deviates from the original $P_{0}$. Thus, consecutive workdays of the same workforce cannot be treated as independent trials and results in the need to (1) daily update the probability that a randomly chosen person becomes infected on the ith day, $P_{0}(\mathrm{i})$, and (2) keep track of the infected and uninfected employees. To do so, we separate the workforce into the following disjoint populations. Let $N_{\text {sus }}, N_{a}, N_{s}, N_{r}$, and $N_{d}$ be vectors indexed over the beginning of the $i$ th day at work and representing the numbers of susceptible, asymptomatic, symptomatic, recovered, and deceased employees, respectively. Additionally, let $N_{\text {inf }}$ denote the number of currently infected individuals, $N_{\text {new }}$ denote the number of daily new infections, and $N_{\text {work }}$ the number of people attending work. The following equations must hold for every day $i$ :

$N_{\text {inf }}(i)=N_{\mathrm{s}}(i)+N_{\mathrm{a}}(i)$

$N_{\text {work }}(i)=N_{\text {sus }}(i)+N_{\mathrm{a}}(i)+N_{\mathrm{r}}(i)$.

In order to reflect the daily change to the percentage of infected employees at work, the following recursive algorithm is used. At the end of the $i$ th day, the model returns $P_{1}(i)$, the probability an uninfected employee is infected on that particular day. Since only $N_{\text {sus }}(i)$ employees are able to contract the virus, the expected number of new infections at the end of the day is $N_{\text {new }}(i)=P_{1}(i) N_{\text {sus }}(i)$. The number of currently infected employees is then calculated to be

$N_{\text {inf }}(i)=N_{\text {inf }}(i-1)+N_{\text {new }}(i)-N_{\mathrm{r}}(i)-N_{\mathrm{d}}(i)$, where

$N_{\mathrm{r}}(i)=N_{\mathrm{r}}(i-1)+\beta N_{\text {new }}(i-\tau)$

for $I>\tau>1$ and zero otherwise. The deceased population is similarly defined, using $1-\beta$ instead of $\beta$. Defining

$N_{\mathrm{s}}(i)=N_{\mathrm{s}}(i-1)+(1-a)\left(N_{\text {new }}\left(i-i_{0}\right)-N_{\text {new }}(i-\tau)\right)$

and

$$
\begin{aligned}
N_{\mathrm{a}}(i)=N_{\mathrm{a}}(i-1)+a & \left(N_{\text {new }}\left(i-i_{0}\right)-N_{\text {new }}(i-\tau)\right) \\
+ & \left(N_{\text {new }}(i)-N_{\text {new }}\left(i-i_{0}\right)\right),
\end{aligned}
$$

one can check that $N_{\mathrm{inf}}(i)=N_{\mathrm{s}}(i)+N_{a}(i)$ for all $i$. If $I<1$, the values of the corresponding vectors are zeros.

In order to initialize the model, we set $N_{\text {inf }}(1)=N_{\text {new }}(1)=P_{0}(1)$ $N_{\text {work }}(1)$, the expected number of infected employees at the beginning of the first day. Note that the range of the expected value of $N_{\text {inf }}(i)$ is continuous, i.e., it may not be a whole number. After using the aforementioned equations to update the population pools, the percentage of infected employees on the $(I+1)$ th day is given by

$$
P_{0}(i+1)=\frac{N_{\mathrm{a}}(i+1)}{N_{\text {work }}(i+1)}
$$

Equation 16 with the given initialization of $N_{\text {new }}$ and Eq. 15 for $N_{a}$ result in the probability of infection equal to $P_{0}(1)$ for all days, if there are no interactions in the workplace. In other words, the model accounts for the probability that employees might be infected outside of the workplace with the probability $P_{0}(1)$, while the interactions at the workplace may result in higher probabilities. Using this process, the model can be applied to multiple workdays to predict the total number of infected employees.

\section{TYPICAL OFFICE EXAMPLE AND A REAL-WORLD CASE}

This section provides an example of model application to a typical office workplace and describes an application of the developed model to a real-world case. The floorplan of a simulated workplace is shown in Fig. 3, with 5 identical floors, and, by assumption, no employee interactions between different floors except for the elevator use and cafeteria.

As one can see in Fig. 3, this floorplan can accommodate 6 people in single offices, 6 people in double-occupancy offices, 8 employees in cubicles, resulting in 20 employees per floor and a 100 -employee building. There are two restrooms on a single floor, being used by 10 employees each, and one elevator in the building. A typical workday includes 2 rides in the elevator, with heavy elevator use for $1.5 \mathrm{~h}$ in the morning and evening; 1 


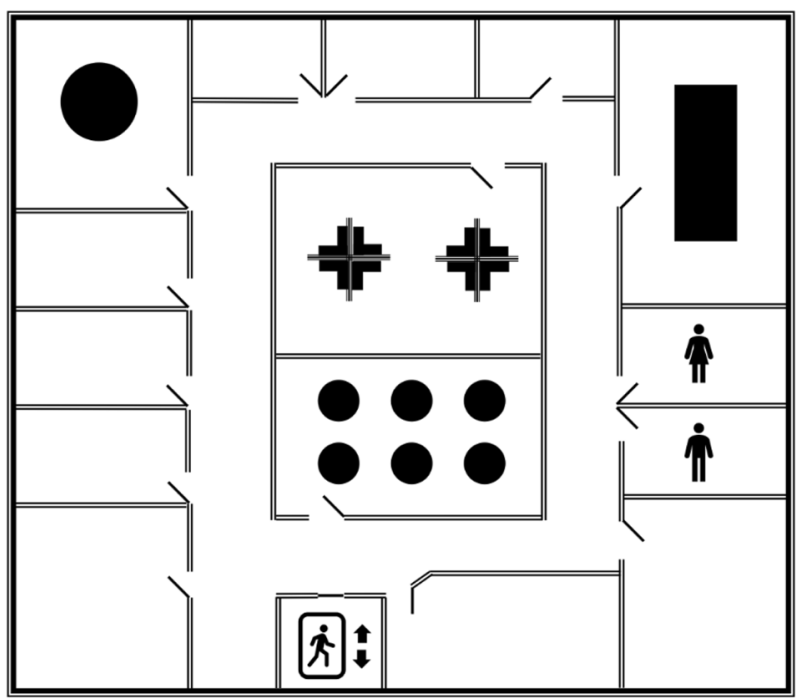

Fig. 3 The floorplan for simulated workplace. Circles denote round tables for work meetings. A rectangle indicates a larger meeting talbe. Female and male pictograms indicate restrooms. Crossshaped tables above six circular tables indicate cubicles, and a walking man pictogram with up and down arrows indicate an elevator. Double ocupancy offices have a larger area than a single occupancy offices.

meeting at a round table with 6 participants; and 1 meeting at a rectangular table with 6 participants. Supplementary Fig. 1 shows probabilities for the restroom and elevator microenvironments, while Supplementary Fig. 2 depicts probabilities at close contacts for $15 \mathrm{~min}$ and $5 \mathrm{~min}$ contact duration.

\section{Parameter choices}

The probability that a random person is infected, $P_{0}$, can be estimated as the ratio of the total number of infected people capable of virus transmission to the total number of people. According to the CDC guidelines, an infected person is capable of virus transmission for about 12-14 days [29]. In our paper, 14 days was used as the infection period, after which an infected person recovers or dies, in accordance to the mortality rate. Therefore, the number of infected people capable of virus transmission can be estimated by summing over all new cases for the past 14 days. Such an estimate can be made on the organizational (workforce), regional, state, or national scale. It also can be refined for specific types of professional occupations, e.g., healthcare workers. For example, on the national scale, the probability that a randomly chosen person in the USA is infected and capable of virus transmission, as of 2 March 2021, can be estimated as:

$P_{0, \text { national }}=\frac{N_{\text {inf }, 14}}{N_{\mathrm{n}}}=\frac{905,961}{330,111,838}=0.0027$,

where $N_{\text {inf, } 14}=905,961$ is the total number of new infections in the USA for the past 14 days, including 2 March 2021 [29], and $N_{\mathrm{n}}$ $=330,111,838$ is the total USA population [30]. In this paper, an infected person implies a person capable of virus transmission.

The percentage of asymptomatic cases among infected was estimated to be about $30 \%$ in early stages of pandemic [31], however, a more recent meta-analysis of various data yielded $17 \%$ [32], echoing by the case study on the Diamond Princess cruise ship, in the confined environment, yielding 18\% [33], which was used in our simulations. The estimates of the incubation period are more consistent and lie in the $4-5$ days range;[34-36] 4 days was used in our simulations. The death rate for the USA, according to the John Hopkins University, is 1.8\% [37]. A complete list of parameters used in this example and their values are shown in the
Supplementary Table 1, where $N(\mu, \sigma)$ denotes the normal distribution with mean $\mu$ and standard deviation $\sigma$.

\section{Effectiveness of pandemic-mitigating policies}

The probability of becoming infected after a workday can be used as a metric to compare the effectiveness of different pandemicmitigating policies. For this example, we consider a few simple restrictive policies in addition to mask mandates.

- Cafeteria Policy-Everyone eats lunch in their office, cafeteria is not used.

- Elevator Policy-Maximum elevator capacity is one.

- Restroom Policy-Maximum restroom capacity is one.

- Office Policy-Everyone has a single office, no cubicle or multi-user office use.

- Meeting Policy-There are no meetings.

- Hallway Policy-If people are encountered in hallways, the closest distance of contact must be substantially larger than $2 \mathrm{~m}$.

The effectiveness of these policies were measured in the percentage of the decrease of daily probability of becoming infected, in comparison with nominal workplace policies.

Figure 4 addresses the efficacy of workplace policy interventions. A single bar shows the reduction in the daily risk of infection as a percentage of the unrestricted case. One can see that the order of most efficient policies is different for the cases of all wearing masks vs. no one wearing masks. Imposing more than one policy is predictably more effective than a single policy. In the case of all employees wearing masks, the combination of cafeteria and meetings policies reduces the chance to get infected by $80 \%$. In the case of no employees wearing masks, several combinations of policies have similar $80 \%$ risk reduction: meetings and office policy, meetings and restrooms, meetings and elevators, meetings and cafeteria, and meetings and hallways. Interestingly, the single most effective policy in both cases is the meeting policy.

\section{Time dynamics simulation}

The time dynamics for the unrestricted workplace is shown in Fig. 5, which provides several insights for the course of pandemic at a workplace. The parameters for the simulation are provided in Supplementary Table 1. The curve depicting currently infected has an exponential growth at the beginning, reaches the full workplace capacity at approximately day 11 , and then, at day 18 , starts receding because the infected population either recovers or deceases. This observation is supported by the recovered and deceased curves, showing growth at the same time as the number of currently infected declines. The number of symptomatic infections peaks at about day 15 , and is delayed in comparison with the total infected curve, because, once an employee becomes infected, he or she remains asymptomatic for a number of days, and then, either becomes symptomatic and is quarantined at home, or remains asymptomatic and continue going to work, or recovers, or deceases. The asymptomatic curve has rather a nontrivial behavior. At the beginning of the pandemic, the asymptomatic infection rapidly spreads and reaches a peak of 15 employees at day 9. Then, it declines mostly due to asymptomatic cases becoming symptomatic. At this time, it coincides with the population going to work, because asymptomatic employees are not aware about their infection and continue their work schedule as usual. Then, after day 15 , the recovery population start increasing, and that decreases both, the symptomatic and asymptomatic cases. Eventually, almost all employees are recovered except those who deceased. The population at work has a remarkable behavior. It has a pronounced minimum of employees at work of about 4 people ( $20 \%$ of the workforce) at day 16 , and then increases to almost the full capacity by day 25 . Note that such a short pandemic lifecycle is in major part due to small number of employees considered in the 
(a)

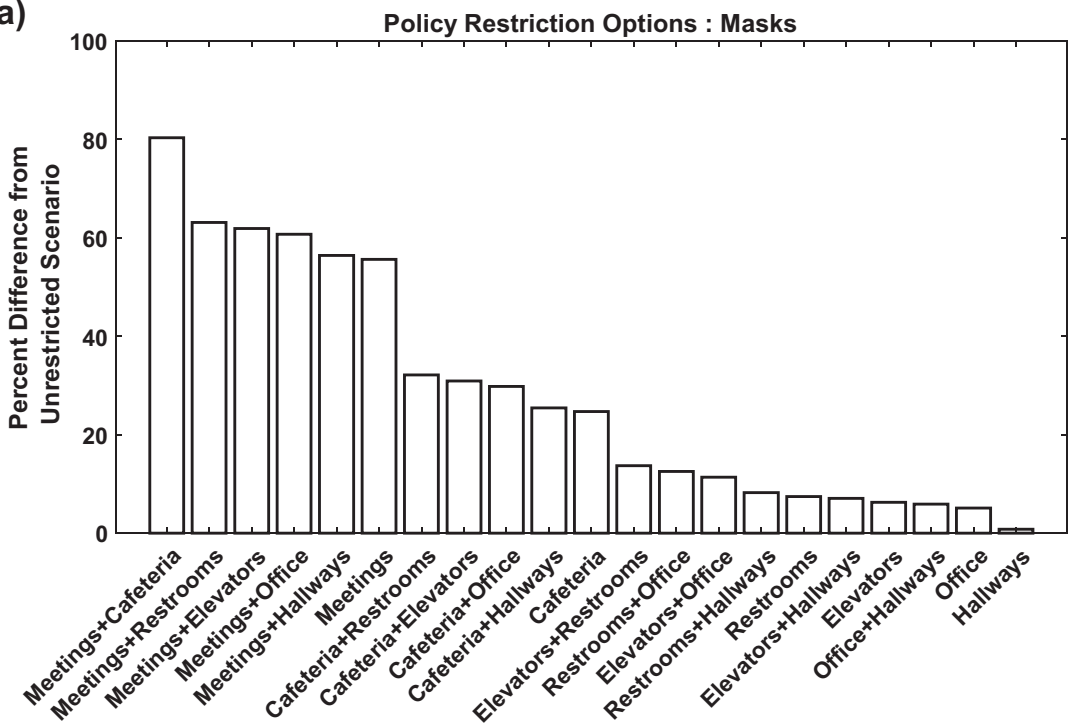

(b)

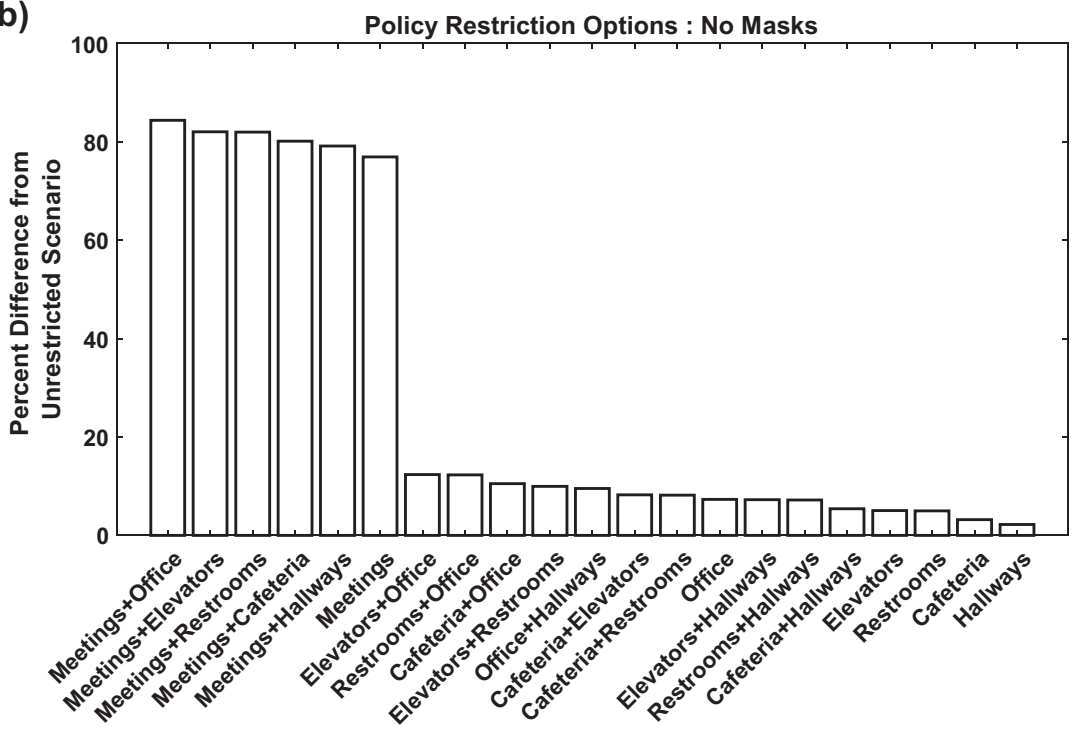

Fig. 4 Relative efficiency of restriction policies. a All wear masks. b No masks.

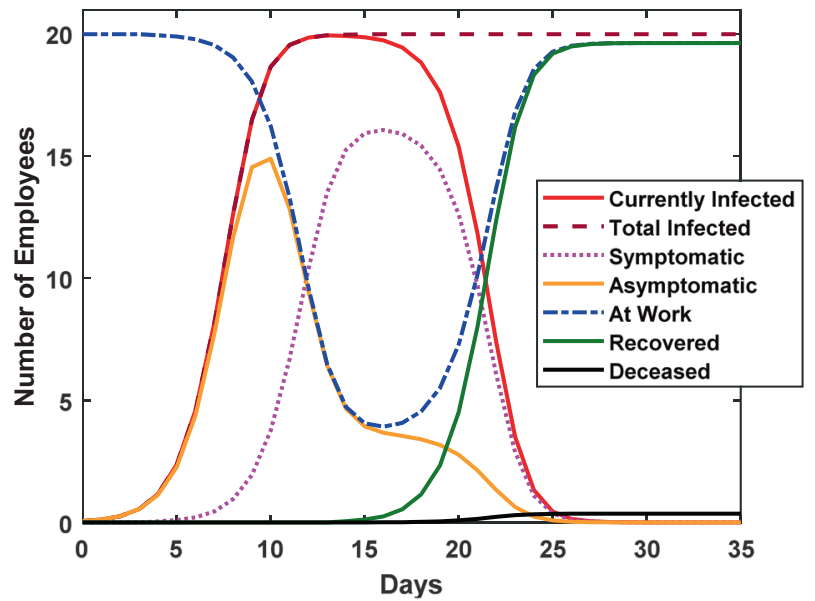

Fig. 5 Expected total number of infected employees when there are no restriction policies. example. For larger workplaces, a different time dynamics is expected.

\section{South Korea call center case}

The example considered above uses national parameters pertinent to the USA. Next, we show applicability of the model to a real case of COVID-19 pandemic outbreak, which was carefully documented at the telephone call center in Seoul Metropolitan area, South Korea [38]. The model parameters of any particular workplace may have deviations from the national or regional statistics. Therefore, the governing parameters of the model should be fitted to empirical data of the specific workplace if the comparison between a model and any particular case to be made.

The working environment for the call center represents an open space with operators seating at large tables. The study represents early stages of pandemic at the call center. New cases of COVID-19 were documented at the center, but all employees were required to attend the work regardless whether they had symptoms or not. The study was terminated at day 14 after 76 employees became infected. In our model, we set that masks were not worn, and all infected were treated as asymptomatic. The daily typical activity was 


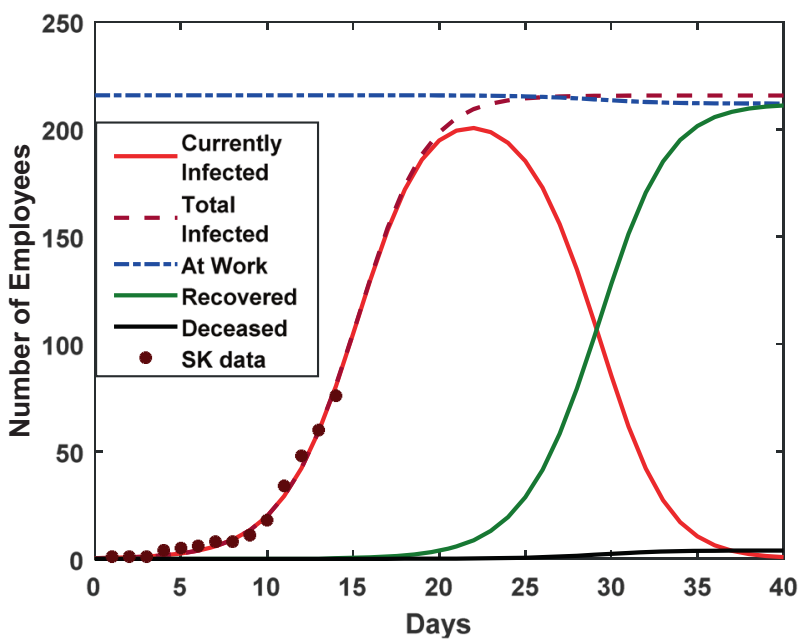

Fig. 6 Expected numbers of infected, recovered, and at work employees for the South Korea case study.

assumed to use the elevator twice a day, bathrooms a few times, and working at the tables. No hallways encounters, cafeteria use, and conference meetings were assumed. The probabilities $P_{0}$ and $P\left(\exp \mid \bar{a}_{i}\right)$ were the fitting parameters to the new registered cases of infection. The complete list of parameters used for the South Korea call center case can be found in Supplementary Table 2 .

The empirical data and model predictions for the South Korea call center case are shown in Fig. 6 . The currently infected curve coincides with the total infected curve up until the infection period ends. After which, people either recover or die, in accordance to the mortality rate. One can see that the exponential growth at the beginning of the pandemic, reflected in the empirical data, is captured accurately by the infection curves. If the call center were not closed, the model predicts that the peak of infections at work would reach almost the entire workforce by the day 20, after which, people would start recovering. However, the total number of infected would still grow so that all people in the center would become infected by the day 25 . Eventually, by day 40 , almost all workforce would recover except for those who might have been deceased.

This example demonstrates that the model can describe realworld cases, provided that the model parameters are fitted to the empirical data.

\section{CONCLUSIONS}

This paper presents a decision-making model suitable for risk assessment and policy evaluation at a workplace regarding COVID-19 infection. The model was applied to a small group within a larger population, accounting for the daily routines of each employee and workplace settings. Yet, this model requires a relatively small number of parameters, and is flexible enough to accommodate uncertainties about COVID-19 transmission mechanisms. The model also accurately reproduced a real-world case study, after being fit to the initial data on new registered cases.

The model combines agent-based, microexposure, and probabilistic modeling principles, making it more robust and addressing uncertainty about the virus being modeled. Unlike previously explored models, the model works through probabilistic estimates of spreading and contracting the disease at various microenvironments directly, with the spatial and temporal virus contraction probability being the modeled quantity of interest. Any contact has non-zero probability of becoming infected, which depends on the duration and the distance of the contact. Such an approach allows one to work with highly uncertain or unknown disease characteristics, increases robustness to the uncertainties in the initial conditions, does not require specific disease spreading parameters or even mechanisms. Unlike agent-based approaches, there is no need to simulate every interaction between agents, and unlike SIR-based approaches, no exact values for the disease transmission rates are required. The model can also be used to estimate time dynamics of the expected total number of infected at a workplace.

The model is also applicable to evaluation of the efficacy of specific disease mitigation policies at a workplace. Simulations of specific scenarios considered in the paper demonstrated that the expected number of new infections for in-person meetings grows cubically with the number of participants, while other microenvironments exhibit quadratic trends, at least, within the considered ranges of probabilities.

The policy that had the greatest influence on infection risk was the mask policy, and for this reason mask use policies were considered separately in each example. The other most risky factor, according to the model, were in-person meetings. The restrictions on both, meetings and cafeteria, meetings and elevators, and meetings and restrooms have the largest effect in risk mitigation, accounting for about $80 \%$ of the total risk.

\section{REFERENCES}

1. Haleem A, Javaid M, Vaishya R. Effects of COVID 19 pandemic in daily life. Curr Med Res. Pract. 2020;10:78-9.

2. Fernandes N. Economic effects of coronavirus outbreak (COVID-19) on the world economy. SSRN Electron J. 2020;1:1-33.

3. Kano T, Yasui K, Mikami T, Asally M, Ishiguro A. An agent-based model of the interrelation between the COVID-19 outbreak and economic activities. Proc R Soc A Math Phys Eng Sci. 2021;477:20200604.

4. Atalan A. Is the lockdown important to prevent the COVID-19 pandemic? Effects on psychology, environment and economy-perspective. Ann Med Surg. 2020;56:38-42.

5. Linkov I, Keenan JM, Trump BD COVID-19: systemic risk and resilience. Springer; New York 2021.

6. Kermack WO, McKendrick AG. A contribution to the mathematical theory of epidemics. Proc R Soc Lond Ser A Containing Pap A Math Phys Character. 1927;115:700-21.

7. Kermack WO, McKendrick AG. Contributions to the mathematical theory of epidemics-II. The problem of endemicity. Bull Math Biol. 1991;53:57-87.

8. Kermack WO, McKendrick AG. Contributions to the mathematical theory of epidemics-III. Further studies of the problem of endemicity. Bull Math Biol. 1991;53:89-118.

9. Calvetti D, Hoover A, Rose J, Somersalo E. Bayesian dynamical estimation of the parameters of an SE (A) IR COVID-19 spread model. 2020. https://arxiv.org/abs/ 2005.04365.

10. Weissman GE, Crane-Droesch A, Chivers C, Luong T, Hanish A, Levy MZ, et al. Locally informed simulation to predict hospital capacity needs during the COVID19 pandemic. Ann Intern Med. 2020;173:21-8.

11. Clancy D, O'Neill PD. Bayesian estimation of the basic reproduction number in stochastic epidemic models. Bayesian Anal. 2008;3:737-57.

12. Chen Y-C, Lu P-E, Chang C-S A Time-dependent SIR model for COVID-19. 2020. https://arxiv.org/abs/2003.00122.

13. Tolles J, Luong T. Modeling epidemics with compartmental models. JAMA. 2020;323:2515-6.

14. Cuevas E. An agent-based model to evaluate the COVID-19 transmission risks in facilities. Comput Biol Med. 2020;121:103827.

15. Silva PCL, Batista PVC, Lima HS, Alves MA, Guimarães FG, Silva RCP. COVID-ABS: An agent-based model of COVID-19 epidemic to simulate health and economic effects of social distancing interventions. Chaos Solitons Fractals. 2020;139:110088.

16. Manzo G, Matthews T. Potentialities and limitations of agent-based simulations. Rev française sociol. 2014;55:653-88.

17. Troitzsch KG. Analysing Simulation Results Statistically: Does Significance Matter? In: Interdisciplinary applications of agent-based social simulation and modeling, edited by Diana Francisca Adamatti, et al., Hershey, PA, IGI Global; 2014, p. 88-105.

18. Hussein T, Löndahl J, Thuresson S, Alsved M, Al-Hunaiti A, Saksela K, et al. Indoor model simulation for COVID-19 transport and exposure. Int J Environ Res Public Health. 2021;18:2927. 
19. Lelieveld J, Helleis F, Borrmann S, Cheng Y, Drewnick F, Haug G, et al. Model calculations of aerosol transmission and infection risk of COVID-19 in indoor environments. Int. J. Environ. Res. Public Health; 2020. p. 1-18.

20. Vuorinen V, Aarnio M, Alava M, Alopaeus V, Atanasova N, Auvinen M, et al. Modelling aerosol transport and virus exposure with numerical simulations in relation to SARS-CoV-2 transmission by inhalation indoors. Saf Sci. 2020;130:104866.

21. Lewis D. Is the coronavirus airborne? Experts can't agree. Nature 2020;580:175.

22. Price PS, Curry CL, Goodrum PE, Gray MN, McCrodden Jl, Harrington NW, et al. Monte Carlo modeling of time-dependent exposures using a microexposure event approach. Risk Anal. 1996;16:339-48.

23. Price PS, Chaisson CF. A conceptual framework for modeling aggregate and cumulative exposures to chemicals. J Expo Sci Environ Epidemiol. 2005;15:473-81.

24. McCarthy JE, Dumas BA, McCarthy MT, Dewitt BD. A deterministic linear infection model to inform Risk-Cost-Benefit Analysis of activities during the SARS-CoV-2 pandemic. 2020. https://www.medrxiv.org/content/10.1101/2020.08.23.20180349v1.

25. Chu DK, Akl EA, Duda S, Solo $K$, Yaacoub $S$, Schünemann HJ, et al. Physical distancing, face masks, and eye protection to prevent person-to-person transmission of SARS-CoV-2 and COVID-19: a systematic review and meta-analysis. Lancet. 2020;395:1973-87.

26. Mueller AV, Eden MJ, Oakes JM, Bellini C, Fernandez LA. Quantitative method for comparative assessment of particle removal efficiency of fabric masks as alternatives to standard surgical masks for PPE. Matter. 2020;3:950-62.

27. Bhagat RK, Wykes MD, Dalziel SB, Linden P. Effects of ventilation on the indoor spread of COVID-19. J Fluid Mech. 2020;903:F1.

28. Sze To GN, Chao CYH. Review and comparison between the Wells-Riley and dose-response approaches to risk assessment of infectious respiratory diseases. Indoor Air. 2010;20:2-16.

29. @CDC. COVID Data Tracker - Centers for Disease Control and Prevention: @CDC; 2021. https://covid.cdc.gov/covid-data-tracker/\#datatracker-h.

30. @Census. U.S. and World Population Clock - The United States Census Bureau: @Census; 2021. https://www.census.gov/popclock.

31. Nishiura H, Kobayashi T, Miyama T, Suzuki A, Jung SM, Hayashi K, et al. Estimation of the asymptomatic ratio of novel coronavirus infections (COVID-19). Int J Infect Dis. 2020;94:154.

32. Byambasuren O, Cardona M, Bell K, Clark J, McLaws M-L, Glasziou P. Estimating the extent of asymptomatic COVID-19 and its potential for community transmission: systematic review and meta-analysis. Off J Assoc Med Microbiol Infect Dis Can. 2020;5:223-34.

33. Mizumoto K, Kagaya K, Zarebski A, Chowell G. Estimating the asymptomatic proportion of coronavirus disease 2019 (COVID-19) cases on board the Diamond Princess cruise ship, Yokohama, Japan, 2020. Eurosurveillance. 2020;25:2000180.

34. Lauer SA, Grantz KH, Bi Q, Jones FK, Zheng Q, Meredith HR, et al. The incubation period of coronavirus disease 2019 (COVID-19) from publicly reported confirmed cases: estimation and application. Ann Intern Med. 2020;172:577-82.
35. Guan W-J, Ni Z-Y, Hu Y, Liang W-H, Ou C-Q, He J-X, et al. Clinical characteristics of coronavirus disease 2019 in China. N Engl J Med. 2020;382:1708-20.

36. Li Q, Guan X, Wu P, Wang X, Zhou L, Tong Y, et al. Early transmission dynamics in Wuhan, China, of novel coronavirus-infected pneumonia. N Engl J Med. 2020;382:1199-207.

37. @JohnsHopkins. Mortality analyses - Johns Hopkins Coronavirus Resource Center: @JohnsHopkins; 2021. https://coronavirus.jhu.edu/data/mortality.

38. Park SY, Kim Y-M, Yi S, Lee S, Na B-J, Kim CB, et al. Coronavirus disease outbreak in call center, South Korea. Emerg Infect Dis. 2020;26:1666.

\section{ACKNOWLEDGEMENTS}

We are grateful to Dr. Paul Price, US EPA, for his help in consultations and explanations of the chemical microexposure model and in reviewing an early version of this manuscript. This paper is based upon work supported by the US Army Engineer Research and Development Center FLEX-4 Project on Systemic Resilience. The views and opinions expressed in this article are those of the individual authors and not those of the US Army or other sponsor organizations.

\section{AUTHOR CONTRIBUTIONS}

SV and IL framed problem and developed a mathematical and statistical modeling framework. DC implemented strawman model and collected data. EW, AM, JD and KD collected data and implemented model as software tool. MK provided senior guidance on modeling and data analytics. BT provided advice on public health issues. All co-authors contributed to paper preparation.

\section{COMPETING INTERESTS}

The authors declare no competing interests.

\section{ADDITIONAL INFORMATION}

Supplementary information The online version contains supplementary material available at https://doi.org/10.1038/s41370-022-00411-2.

Correspondence and requests for materials should be addressed to Sergey Vecherin or Igor Linkov.

Reprints and permission information is available at http://www.nature.com/ reprints

Publisher's note Springer Nature remains neutral with regard to jurisdictional claims in published maps and institutional affiliations. 